\title{
"METODOLOGIA DE ELABORAÇÃO DA CARTA DO POTENCIAL EROSIVO DA BACIA DO RIO DAS VELHAS (MG)"
}

\author{
Britaldo Silveira Soares Filho(*), Valéria Amorim do Carmo(**) \& Wagner J. Nogueira(***)
}

\begin{abstract}
This paper concerns the methodology applied to the erosive potential analysis in the Rio da Velhas hydrographic basin (Minas Gerais State, Brasil) along with the results obtained. This project aimed at the production of a digital map at a 1: 200000 scale of the fragility of both natural and human-interfered environments in Rio das Velhas Basin. The adopted approach seeks to integrate the combined effects of a set of variables - relief, soil, climate and land use/land cover - to identify the environmental instability and to map the ecodynamic units. First, the cartographic variables were classified according to their degree of environmental instability, these were later combined pair-wise and a set of decision rules was applied to produce the final map. The results depicted well the major fragile areas in the basin, which should receive special care for their preservation. The developed methodology is easily implemented and flexible enough to permit changing the decision rules to fit different criteria.
\end{abstract}

\section{INTRODUÇÃO}

O presente artigo reporta a metodologia desenvolvida e os resultados obtidos da análise do potencial erosivo na Bacia Hidrográfica do Rio das Velhas (MG). O projeto em questão teve por objetivo a elaboração de uma carta, em meio digital, compatível com a escala 1: 200 000, da fragilidade dos ambientes naturais e antropizados da Bacia do Rio das Velhas, denominada aqui neste trabalho como carta do potencial erosivo.

Este estudo baseia-se nas idéias desenvolvidas por Tricart (1977) e na metodologia adaptada por Ross (1992), a qual visa identificar, a partir de um conjunto de variáveis ambientais, o grau de estabilidade/ instabilidade das unidades ecodinâmicas. Segundo Tricart (1977), as unidades ecodinâmicas, compostas por elementos do meio biótipo e físico e constituindo um sistema ambiental de troca de energia e matéria, podem se encontrar em vários estágios de equilíbrio dinâmico, o qual pode ser alterado pela intervenção antrópica, levando a uma situação de instabilidade geomorfológica. Como resultado dessa instabilidade, ocorre erosão acentuada, com a perda do solo, degradação ambiental e conseqüente assoreamento das bacias fluviais a jusante. Nesses termos, Ross (1992) define as unidades ecodinâmicas instáveis como sendo aquelas cujas intervenções antrópicas modificaram intensamente os ambientes naturais através do desmatamento e práticas de diversas atividades econômicas. O mesmo autor classifica essas unidades de acordo com o seu grau de instabilidade em: instabilidade emergente, instabilidade muito fraca à instabilidade muito forte. Por outro lado, as unidades ecodinâmicas estáveis são as que se encontram em seu estado natural e estão em equilíbrio dinâmico, logo, poupadas da ação humana. Ainda, algumas classes de unidades ecodinâmicas estáveis, apesar de estarem em equilíbrio dinâmico, podem apresentar uma instabilidade potencial previsível diante de suas características naturais e possibilidade de inserção antrópica (Ross, 1992).

Portanto, a carta de fragilidade ambiental ou potencial erosivo consiste em um fundamental instrumento ao planejamento físico-territorial, para fins de destacar importantes e frágeis ecossistemas regionais, como também na identificação de áreas que necessitem de uma intervenção para se mitigar ocorrentes problemas ambientais. Por isso, serão descritos a seguir os principais passos e procedimentos metodológicos empregados para a obtenção da mesma.

\section{METODOLOGIA DE ANÁLISE}

Hopkins (1977) discorre sobre as diversas abordagens que podem ser usadas para obtenção de mapas de padrões espaciais, formados pelos requerimentos preferenciais à ocorrência de um certo fenômeno ou condição ambiental, com no exemplo de uma carta de fragilidade ambiental. Segundo este autor, o melhor procedimento para obtenção de tais mapas, na maioria dos casos, consiste em se usar métodos de combinação linear e não linear em um primeiro estágio, para serem então seguidos, em uma segunda fase, por regras de combinação. Ou seja, num primeiro passo incorporam-se os relacionamentos entre os fatores, cujas relações matemáticas são conhecidas, e em seguida, combinam-se de modo hierárquico, outros fatores, que não podem ser expressos por relações matemáticas, através de uma ordenação de adequação, de acordo com um processo de regras de decisão, o qual pode ser 
derivado de medidas de campo, ou mesmo arbitrado por uma visão imposta pelos especialistas escolhidos para estudar a questão.

Em efeito, é através dessa abordagem que este trabalho busca integrar a influência de um conjunto de variáveis ambientais, a saber: relevo, solo, clima e o uso e cobertura do solo para determinar as diferentes unidades ecodinâmicas. A metodologia de análise espacial visa, numa primeira instância, categorizar esses dados cartográficos, ordenando-os em função de suas influências em relação à fragilidade ambiental, para numa segunda etapa, utilizar regras de cruzamento, que adicionam, a cada passo, o efeito combinado de cada uma dessas variáveis em um mapa composto até se chegar no mapa final do potencial erosivo, o qual integra a influência conjunta de todas as variáveis. Como resultado desse cruzamento espacial, são identificadas manchas de diferentes padrões de fragilidade, as quais são hierarquizadas em categorias, que vão desde as unidades ecodinâmicas estáveis, incluindo as de instabilidade potencial, ou seja, aquelas que uma intervenção antrópica poderá resultar em uma instabilidade e aceleração dos processos morfodinâmicos, até as unidades ecodinâmicas instáveis.

Para a implementação dessa análise é usado um Sistema de Informações Geográficas, o qual pode ser conceituado como um conjunto de facilidades voltado à captura, armazenamento, verificação, integração, manipulação, análise e visualização de dados referenciados à Terra (Taylor, 1991). Nesse ambiente, o procedimento analítico utilizado envolve o processamento cíclico de mapas digitais (semelhante à resolução de parênteses de uma equação), usando-se de linguagens e operadores de álgebra de mapas (Cf. Tomlin, 1990 e Berry, 1993). Desse modo, uma seqüência lógica, coerente para o desempenho da análise cartográfica em questão, envolve a recuperação de um ou mais mapas da base de dados, processamento desses dados como especificado pelo usuário, criação de um novo mapa contendo o resultado do processamento e o seu armazenamento para o processamento subseqüente. Assim, serão descritos a seguir o tratamento de dados e as regras utilizadas em cada etapa de obtenção da carta de potencial erosivo.

\section{A CARTA DE DECLIVIDADE}

A influência do relevo, em um estudo de fragilidade ambiental, pode ser computada através do índice de dissecação - relação entre a densidade de drenagem e o entalhamento dos canais fluviais -, sendo este índice usado em geral para escalas 1: 50000 ou menores, ou através das classes de declividade, neste caso para escalas 1: 25000 ou maiores (Ross, 1992). No presente estudo, como ambas as variáveis são correlatas (Fig.1), foi utilizada a variável declividade derivada do modelo digital do terreno obtido da conversão e processamento digital dos mapas topográficos na escala 1:50 $000 \mathrm{e}$ 1:100 000. O primeiro passo neste processo consistiu na geração de um modelo em rede de triângulos irregulares, usando-se da partição de Delauney (Burrough, 1991). Em seguida, esta representação da superfície topográfica foi convertida numa grade regular com resolução de 50 metros, por meio de um algoritmo de interpolação que seleciona os dois pontos mais próximos por quadrante e pondera os valores $\mathrm{Z}$ pelo inverso do quadrado da distância (Fig. 2). Deste modelo, foi derivado o mapa de declividade, utilizando o seguinte operador gradiente: $\tilde{N}(x, y)=\left[\left(\tan _{x}\right)^{2}+\right.$ $\left.\left(\tan q_{y}\right)^{2}\right]^{1 / 2}$. Onde $q_{x}$ e $q_{y}$ são, respectivamente, os ângulos na direção x e y da superfície formada pelos valores dos Zs das células vizinhas (Monmonier, 1982). Por último, o mapa digital de declividade, com valores em graus, foi classificado em 5 classes de acordo com o definido por Ross $(1992,1996)$ Vide Tab. 1 e Fig. 5a.

\section{Densidade de Drenagem}

Entalhamento

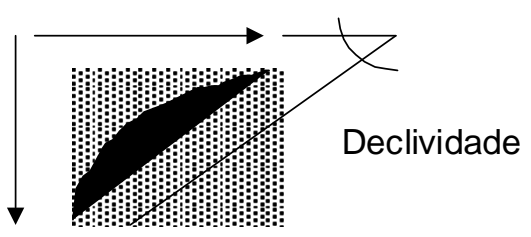

Figura 1: Relação entre a declividade e o índice de dissecação dado pelo cruzamento entre o grau de entalhamento e a dimensão interfluvial.

Figure 1: Relation between the slope and the dissecation index.

\begin{tabular}{|c|c|c|c|}
\hline Categorias & $\%$ & Graus & Legenda \\
\hline Muito Fraca & $0 \%-6 \%$ & $0-5.14$ & $\mathbf{1}$ \\
\hline Fraca & $6 \%-12 \%$ & $5.14-10.8$ & $\mathbf{2}$ \\
\hline Média & $12 \%-20 \%$ & $10.8-18$ & $\mathbf{3}$ \\
\hline Forte & $20 \%-30 \%$ & $18-27$ & $\mathbf{4}$ \\
\hline Muito Forte & $>30 \%$ & $>27$ & $\mathbf{5}$ \\
\hline
\end{tabular}

Tabela 1: Classes de declividade.

Table 1: Slope classes. 




Figura 2: Mapa de relevo sombreado obtido do modelo digital de terreno da Bacia do Rio das Velhas. Figure 2: Shaded relief map derived from the digital terrain model. 


\section{A CARTA DE ERODIBILIDADE}

O mapa de solos é a segunda variável empregada neste estudo. Como fonte desse dado foi utilizado o mapa de solos, na escala 1: 200 000, elaborado pelo CETEC/MG. Segundo Ross (1992), os graus de erodibilidade dos solos, considerando o escoamento superficial difuso e concentrado das águas pluviais, podem ser agrupados em 5 classes. Tendo, portanto, como base essa classificação, o mapa dos tipos de solos ocorrentes na região, após conversão para meio digital ${ }^{1}$, foi reclassificado de acordo com os graus de erodibilidade (Tab. 2 e Fig. 5b). polígonos de Voronoi. A carta resultante foi então reclassificada em 3 classes de $R$ (Fig. 3 e 5c). Esta classificação foi gerada com base na média dos dados e o desvio padrão, sendo, em resultado, a classe $u m$ equivalente aos valores menores que a média menos 1 desvio padrão $(672,02)$, a classe dois corresponde aos valores entre a média e -1 e +1 desvio padrão $(672,02-781,18)$ e por último, a classe três abrange os valores acima da média +1 desvio padrão (781,18). A Fig. 3 mostra que esses limiares correspondem bem a estrutura de distribuição dos valores de $R$.

\begin{tabular}{|cl|l|c|}
\hline $\begin{array}{c}\text { Graus de } \\
\text { Erodibilidade }\end{array}$ & \multicolumn{1}{c|}{ Tipos de Solos } & Cod_solo & Legenda \\
\hline Muito Baixa & Latossolo Vermelho Escuro & LEa1,LEa2,LEd1,Lva1 & $\mathbf{1}$ \\
\hline Baixa & Latossolo Vermelho Amarelo & Lva2,LVa3,LVd1,LVd2,LVd3 & $\mathbf{2}$ \\
\hline Média & Podzólico Vermelho Amarelo & PV,PE & $\mathbf{3}$ \\
\hline Alta & Cambissolos, Solos Aluviais & Ad1,Ae1,Ae2,Ca,Cd1,Cd2,Cd3,Ce & $\mathbf{4}$ \\
\hline Muito Alta & $\begin{array}{l}\text { Solos Litólicos, Areias Quartzosa e } \\
\text { Afloramento de Rochas }\end{array}$ & AQ, Aqa, AR1,Rd,Re & $\mathbf{5}$ \\
\hline
\end{tabular}

Tabela 2 : Classes de erodibilidade por tipo de solo.

Table 2: Erodibility soil classes.

\section{A CARTA DE EROSIVIDADE}

A próxima variável a entrar no modelo se faz representar pela erosividade das chuvas, ou fator $R$ ou $E$ da equação universal de perda dos solos. Segundo Jansson (1982), o cálculo da erosividade pela chuva é essencial para qualquer previsão da erosão. Para tanto, um parâmetro encontrado que apresentou o melhor resultado sob diferentes condições de precipitação e tipos de solo foi o $E I_{30}$ (Wichmeier \& Smith, 1958 apud El-Swaify et al., 1982), o qual equivale à energia cinética produzida por uma tempestade em um período de 30 minutos. De acordo com Jansson (1982), um índice que pode ser correlacionado com o $E I_{30}$ é uma modificação do índice de Fournier, dada por:

$$
\sum_{i=1}^{12} p_{i}{ }^{2} / P \text { onde } p_{i} \text { é a precipitação mensal e } P \text { é }
$$

Como as constantes da regressão diferem de uma região para outra, foi utilizado neste trabalho os valores calculados por Lombardi Neto \& Moldenhauer (1980) apud Ross (1992).

$R=6,866 .\left(p^{2} / P\right)^{0.85}$ onde $p$ precipitação média mensal e $P$ precipitação média anual.

$\mathrm{O}$ valor $R$ foi então obtido para um número de 27 de estações meteorológicas da Bacia do Rio das Velhas (Tab.3), tendo sido calculadas as médias mensais e anuais de uma variada série histórica, cuja a maioria das estações possui dados coletados por mais de 40 anos, outras com cerca de 22 anos e algumas com apenas 10 anos. As áreas de influência das estações foram estabelecidas, usando-se

\begin{tabular}{|c|c|c|c|c|}
\hline $\mathbf{N}^{\mathbf{0}}$ & Estação & $\mathbf{p}^{2} / \mathbf{P}$ & $R$ & Classes \\
\hline 1 & 01844001 & 195,22 & 607,65 & 1 \\
\hline 2 & 01844009 & 207,68 & 640,46 & 1 \\
\hline 3 & 01944020 & 216,24 & 662,83 & 1 \\
\hline 4 & 01943042 & 216,24 & 662,83 & 1 \\
\hline 5 & 01744010 & 222,88 & 680,08 & 2 \\
\hline 6 & 01943049 & 223,83 & 682,55 & 2 \\
\hline 7 & 01844010 & 226,13 & 688,50 & 2 \\
\hline 8 & 01943009 & 230,31 & 699,32 & 2 \\
\hline 9 & 01843002 & 234,24 & 709,44 & 2 \\
\hline 10 & 02043056 & 235,71 & 713,23 & 2 \\
\hline 11 & 01944024 & 236,66 & 715,65 & 2 \\
\hline 12 & 01943004 & 238,21 & 719,64 & 2 \\
\hline 13 & 01843000 & 240,96 & 726,70 & 2 \\
\hline 14 & 02043060 & 241,52 & 728,14 & 2 \\
\hline 15 & 01943049 & 242,74 & 731,27 & 2 \\
\hline 16 & 01943024 & 245,56 & 738,48 & 2 \\
\hline 17 & 01943023 & 245,56 & 738,48 & 2 \\
\hline 18 & 01744030 & 251,59 & 753,86 & 2 \\
\hline 19 & 01844019 & 253,65 & 759,10 & 2 \\
\hline 20 & 01943010 & 254,80 & 762,02 & 2 \\
\hline 21 & 01844018 & 256,63 & 766,68 & 2 \\
\hline 22 & 01943006 & 258,63 & 771,75 & 2 \\
\hline 23 & 01844017 & 260,67 & 776,92 & 2 \\
\hline 24 & 02043002 & 265,10 & 788,13 & 3 \\
\hline 25 & 01943000 & 272,60 & 807,04 & 3 \\
\hline 26 & 01744009 & 294,11 & 860,87 & 3 \\
\hline 27 & 01943022 & 350,75 & 999,88 & 3 \\
\hline
\end{tabular}

Tabela 3: Valores de erosividade para estações da bacia do Rio das Velhas.

Table 3: Erosivity values for climate stations in Rio das Velhas basin. 




Figura 3: Distribuição dos valores de R.

Figure 3: Distribution of $R$ values.

\section{A CARTA DE USO E COBERTURA DO SOLO}

A última variável a ser integrada na análise do potencial erosivo se fez representar pelo mapa de uso e de cobertura do solo. Como nesse caso, houve necessidade de se obter informação cartográfica atualizada, foram utilizadas imagens do satélite TM/ LANDSAT- 5 como principal fonte de dados. As bandas selecionadas foram a $\operatorname{tm} 3(0.66 \mathrm{~mm})$ - vermelho, $t m 4$ $(0.83 \mathrm{~mm})$ - infravermelho próximo e tm5 $(1.65 \mathrm{~mm})$ infravermelho médio. A escolha dessas bandas baseouse no comportamento espectral da vegetação exibido no processo de interação com a REM (Radiação Eletromagnética). Desse modo, a faixa do vermelho mede a concentração de pigmentos foliares, a do infravermelho próximo a densidade de biomassa e a do infravermelho médio a concentração de umidade foliar. Diferentes fisionomias vegetais, em diversos estados fenológicos, se diferenciarão por suas respostas espectrais em uma imagem composta por essas bandas. Já a seleção da data de aquisição dos dados buscou otimizar a obtenção de uma data mais recente, desprovida de nuvens e que correspondesse ao período da estação seca, quando é de se esperar um maior contraste entre as diversas fisionomias vegetais (Tab. 4). Por outro lado, a escolha dessa época de aquisição impossibilitou a identificação das culturas de verão.

A definição das classes de mapeamento baseou-se na potencialidade de discriminação das imagens de satélite e do interesse em produzir os dados necessários ao modelo do potencial erosivo e fragilidade ambiental em questão. Nesse propósito, foram definidas 13 classes de mapeamento, a saber: 1) Mata, 2) Mata Ciliar, 3) Cerrado, 4) Campo Cerrado, 5) Campo Rupestre, 6) Cerrado em Regeneração, 7) Capoeira, 8) Reflorestamento, 9) Agricultura Irrigada, 10) Agricultura de Sequeiro, 11) Pasto, 12) Área Urbana e 13) Mineração. Por conseguinte, esta legenda engloba tanto as classes de cobertura do solo (no sentido de revestimento do solo) como de uso do solo (no sentido do uso cultural dado pelo homem a uma certa cobertura de solo).

O primeiro passo da análise de imagens incluiu o registro geométrico dos dados. Para tanto, cada uma das imagens digitais foi corrigida geometricamente, usando-se como pontos de controle as coordenadas geográficas obtidas diretamente dos arquivos digitais das cartas topográfica, a partir da referência dos cruzamentos de drenagem identificados em ambos os produtos. Como resultado, foram obtidas novas imagens na projeção UTM, SAD69, fuso 23. O erro médio resultante do processo de ajuste polinomial foi da ordem

\begin{tabular}{|c|c|c|c|c|}
\hline ÓRBITA & PONTO & QUADRANTE & BANDAS & DATA \\
\hline 219 & 72 & $\mathrm{~B}$ & $3,4,5$ & $27 / 04 / 96$ \\
\hline 219 & 72 & $\mathrm{D}$ & $3,4,5$ & $27 / 04 / 96$ \\
\hline 218 & 72 & $\mathrm{C}$ & $3,4,5$ & $25 / 07 / 96$ \\
\hline 218 & 73 & - & $3,4,5$ & $25 / 07 / 96$ \\
\hline 218 & 74 & - & $3,4,5$ & $25 / 07 / 96$ \\
\hline \multicolumn{5}{|c}{ Tabela 4: Bases de imagens de satélites. }
\end{tabular}

Table: Satellite database. 
de um pixel, \pm 30 metros, o que atende bem a precisão cartográfica para escala 1: 100000 ou menor. A partir das imagens georreferenciadas, foi gerado um mosaico digital que cobrisse toda a bacia. Neste processo, os histogramas das bordas das junções entre imagens foram comparados e as imagens de 27/04/96 foram transformadas linearmente para se obter um balanço de cor mais homogêneo. As junções entre as diversas imagens foram estabelecidas a partir de limites, desenhados vetorialmente, que permitissem sempre a eliminação das áreas de borda, onde as deformações são maiores. Após a construção do mosaico, foram excluídas as áreas externas à Bacia Hidrográfica do Rio das Velhas. O produto final consistiu então em um mosaico de imagens Landsat-TM, na forma exata da Bacia do Rio das Velhas (Fig. 4).
Devido à grande extensão da área de interesse, foi empregada uma metodologia híbrida de mapeamento, na qual um conjunto de temas foi extraído diretamente por interpretação visual e os restantes obtidos a partir de técnicas de reconhecimento de padrões. Para interpretação visual, foram geradas composições coloridas tm4Rtm3Btm5G com realce por filtragem espacial (passa alto) na banda I (intensidade). Assim, os temas, tais como os diversos tipos de campos, área urbana e mineração, foram interpretados visualmente, tendo como referência chaves de interpretação obtidas de áreas conhecidas. Já os outros temas foram mapeados, em primeiro, usando-se da técnica de classificação de imagens por máxima verossimilhança, seguida de uma edição visual. Como, a classificação por máxima verossimilhança baseia-se nos parâmetros

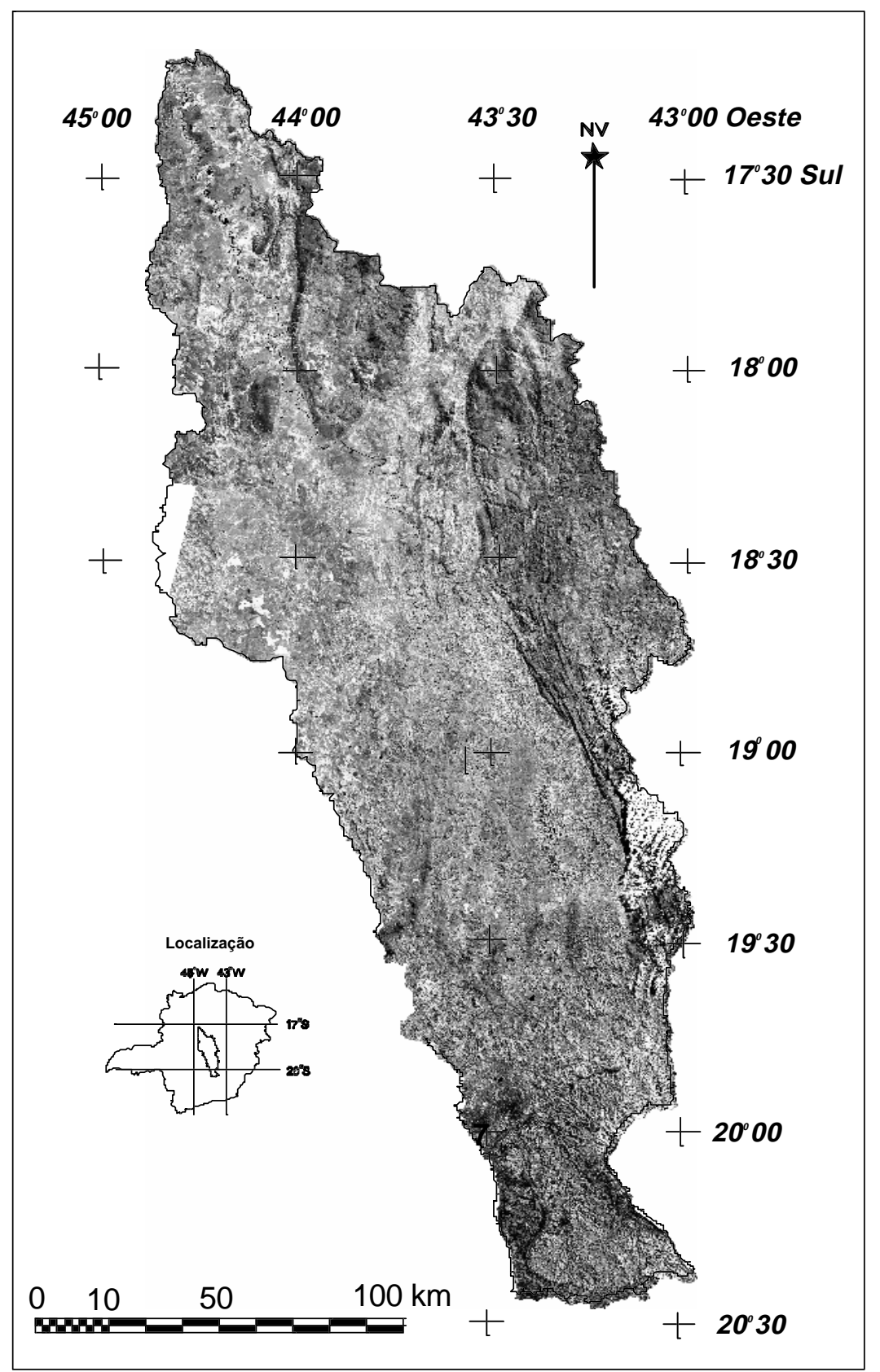

Figura 4: - Mosaico georreferenciado de imagens TM/Landsat-5, banda 4. Figure 4: Georeferenced LANDSAT mosaic of the Rio das Velhas Basin, band tm4. 
estatísticos obtidos para cada classe de mapeamento, foram identificadas áreas de treinamento a partir da experiência prévia de campo e dos mapas de vegetação elaborados pelo IEF/MG.

Para o processo de classificação, foram empregadas as três bandas originais adquiridas e mais o Índice Vegetação por Diferença Normalizada - IVDN = $(\mathrm{TM} 4$ - TM3)/(TM4+TM3). Esta imagem, por ser resultado de uma razão de bandas, mostra uma atenuação da variação de iluminação devido ao relevo, sendo portanto empregada no processo de classificação com uma feição a mais de informação. Ao final do processo de classificação, as diversas classes espectrais (solo exposto, água, sombra, pasto, campo, cerrado, reflorestamento, capoeira, mata, etc.) foram agrupadas para um conjunto de apenas três classes: 1: (mata, reflorestamento e cerrado), 2: (capoeiras), 3: (pastagem e campo). Este procedimento foi realizado para minimizar as imprecisões produzidas pelo processo de classificação. Para fins de generalização, a imagem classificada foi ainda filtrada, usando o filtro da moda $7 \times 7$, e por fim, as pequenas manchas menores que 1 hectare foram eliminadas por meio de um filtro, que testa a conectividade de um pixel central em relação aos vizinhos pertencentes a mesma classe. A imagem final foi então vetorizada e sobreposta à composição colorida para fins de edição, correção e reclassificação para os temas finais de mapeamento, usando-se agora interpretação visual. Desse modo, a classificação de imagens não representou um produto final, mas sim um dado ancilar ao processo de interpretação de imagens.

Após a obtenção do mapa final de uso e cobertura do solo, as treze categorias de mapeamento foram reclassificadas de acordo com o seu grau de proteção ambiental, como proposto por Ross (1992). Vide Tab. 5 e Fig. 5c.

\begin{tabular}{|c|c|c|}
\hline Graus de Proteção & Tipos de Cobertura & Legenda \\
\hline Muito Alto & Mata, Mata Ciliar & $\mathbf{5}$ \\
\hline Alto & Cerrado, Capoeira & $\mathbf{4}$ \\
\hline Médio & Reflorestamento, Campo Cerrado, Cam po Rupestre, & 3 \\
\hline B aixo & Cerrado em Regeneração & $\mathbf{2}$ \\
\hline Muito B aixo a Nulo & Agricultura Irrigada e Sequeiro, Pasto & $\mathbf{1}$ \\
\hline
\end{tabular}

Tabela 5: Graus de Proteção por tipo de Cobertura e Uso do Solo.

Table 5: Degrees of protection for land use/landcover classes.
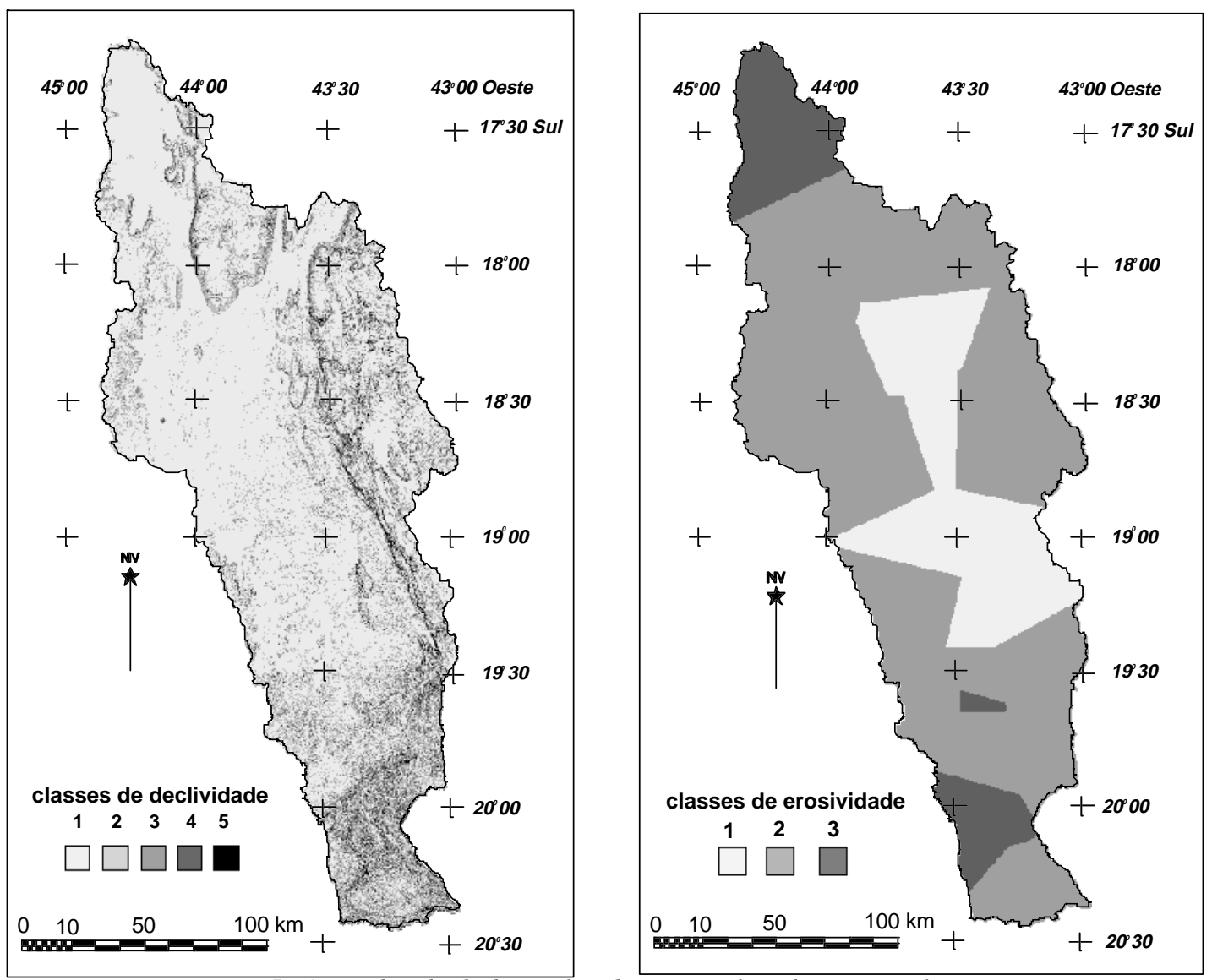

Figure 5: Camadas de dados utilizadas na análise do potencial erosivo.

Figure 5: Cartographic data used in the erosive potential analysis. 

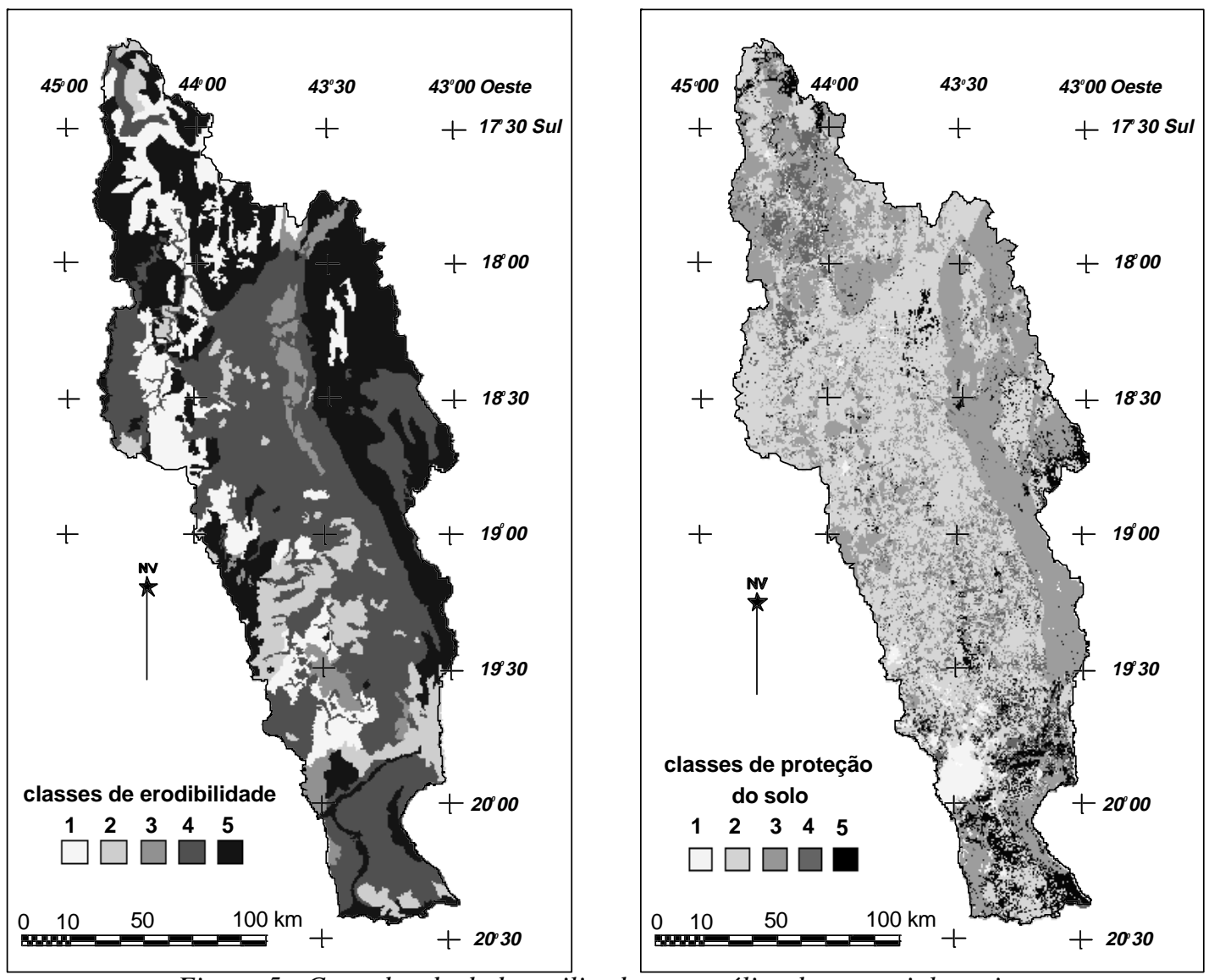

Figura 5: Camadas de dados utilizadas na análise do potencial erosivo.

Figure 5: Cartographic data used in the erosive potential analysis.

\section{A CARTA DO POTENCIAL EROSIVO E INSTABILIDADE AMBIENTAL}

Após a reclassificação das quatro variáveis cartográficas descritas anteriormente. Estas foram cruzadas duas a duas, usando-se de um conjunto de regras de hierarquização. Assim, as classes de erodibilidade e declividade foram combinadas, de acordo com a regra estabelecida pela Tab. 6, dando origem a 5 novas classes (Tab. 7) e um novo mapa denominado como potencial de erodibilidade/ declividade. Este mapa foi então armazenado e cruzado com o mapa de erosividade, através da regra expressa na Tab. 8, resultando em um novo mapa intermediário. Por fim, fez-se o cruzamento deste último com o mapa de grau de proteção ambiental, de acordo com as regras estabelecidas pela Tab. 9. O produto deste processamento resultou então no mapa final de fragilidade das Unidades Ecodinâmicas, no qual são assinaladas as classes, muito estável, estável, potencialmente instável, potencialmente muito instável, instabilidade emergente, instável e muito instável (Tab. 10 e Fig. 6).

Como resultado, o mapa final, representado pela Fig. 6, mostra que as principais áreas de instabilidade são a porção sudeste do Quadrilátero Ferrífero, a região metropolitana de Belo Horizonte, a borda ocidental central da Bacia, a região da cordilheira do Espinhaço, Serra do Cabral e o extremo setentrional da Bacia. Ainda, muitas áreas a sul de Belo Horizonte apresentam uma instabilidade potencial, que é amenizada pela sua expressiva cobertura florestal. Portanto, recomenda-se a preservação dessas áreas que se encontram em um frágil equilíbrio dinâmico.

\begin{tabular}{|c|c|c|c|c|c|}
\hline \multicolumn{7}{|c|}{ Classes de erodibilidade } \\
\hline Declividade & $\mathbf{1}$ & $\mathbf{2}$ & $\mathbf{3}$ & $\mathbf{4}$ & $\mathbf{5}$ \\
\hline $\mathbf{1}$ & $\mathbf{1 1}$ & $\mathbf{1 2}$ & $\mathbf{1 3}$ & 14 & $\mathbf{1 5}$ \\
\hline $\mathbf{2}$ & $\mathbf{2 1}$ & $\mathbf{2 2}$ & $\mathbf{2 3}$ & 24 & $\mathbf{2 5}$ \\
\hline 3 & $\mathbf{3 1}$ & $\mathbf{3 2}$ & $\mathbf{3 3}$ & $\mathbf{3 4}$ & $\mathbf{3 5}$ \\
\hline $\mathbf{4}$ & 41 & 42 & $\mathbf{4 3}$ & $\mathbf{4 4}$ & $\mathbf{4 5}$ \\
\hline $\mathbf{5}$ & $\mathbf{5 1}$ & $\mathbf{5 2}$ & $\mathbf{5 3}$ & $\mathbf{5 4}$ & $\mathbf{5 5}$ \\
\hline
\end{tabular}

Tabela 6: Regras de cruzamento para as variáveis Declividade e Erodibilidade. Table 6: Rule for compositing Slope and Erodiblility. 




Figura 6: Mapa Final do Potencial Erosivo e Instabilidade Ambiental da Bacia do Rio das Velhas. Figure 6: The erosive potential and environmental instability map of Rio das Velhas Basin.

\begin{tabular}{|c|c|}
\hline Classes & Legenda \\
\hline Muito Baixo a Nulo & $\mathbf{1}$ \\
\hline Baixo & $\mathbf{2}$ \\
\hline Médio & $\mathbf{3}$ \\
\hline Alto & $\mathbf{4}$ \\
\hline Muito Alto & $\mathbf{5}$ \\
\hline
\end{tabular}

Tabela 7: Classes do mapa composto declividade lerodibilidade.

Table 7: Classes of the slopelerodibility.

\begin{tabular}{|c|r|r|r|r|r|}
\hline \multicolumn{7}{|c|}{ Potencial erodibilidade/declividade } \\
\hline Erosividade & $\mathbf{1}$ & $\mathbf{2}$ & $\mathbf{3}$ & $\mathbf{4}$ & $\mathbf{5}$ \\
\hline $\mathbf{1}$ & $\mathbf{1 1}$ & $\mathbf{1 2}$ & $\mathbf{1 3}$ & $\mathbf{1 4}$ & $\mathbf{1 5}$ \\
\hline $\mathbf{2}$ & $\mathbf{2 1}$ & $\mathbf{2 2}$ & $\mathbf{2 3}$ & 24 & $\mathbf{2 5}$ \\
\hline $\mathbf{3}$ & $\mathbf{3 1}$ & $\mathbf{3 2}$ & 33 & $\mathbf{3 4}$ & $\mathbf{3 5}$ \\
\hline
\end{tabular}

Tabela 8: Regra de cruzamento para as variáveis Potencial erodibilidade/declividade e erosividade. Table 8: Rule for compositing slope lerodibility potential and erosivity.

2 - Oswaldo Bueno Amorim Filho et al. - "Cidades de Porte Médio e o Programa de Ações Sócio - Educativo Culturais para as Populações Carentes do Meio Urbano em Minas Gerais " Belo Horizonte - 1980. 


\begin{tabular}{|c|r|r|r|r|r|}
\hline & \multicolumn{7}{|c|}{ Potencial erosividade/erodibilidade/declividade } \\
\hline Graus de Proteção & $\mathbf{1}$ & $\mathbf{2}$ & $\mathbf{3}$ & $\mathbf{4}$ & $\mathbf{5}$ \\
\hline $\mathbf{5}$ & $\mathbf{1 1}$ & $\mathbf{1 2}$ & $\mathbf{1 3}$ & $\mathbf{1 4}$ & $\mathbf{1 5}$ \\
\hline $\mathbf{4}$ & $\mathbf{2 1}$ & $\mathbf{2 2}$ & $\mathbf{2 3}$ & $\mathbf{2 4}$ & $\mathbf{2 5}$ \\
\hline $\mathbf{3}$ & $\mathbf{3 1}$ & $\mathbf{3 2}$ & 33 & $\mathbf{3 4}$ & $\mathbf{3 5}$ \\
\hline $\mathbf{2}$ & 41 & 42 & $\mathbf{4 3}$ & $\mathbf{4 4}$ & $\mathbf{4 5}$ \\
\hline $\mathbf{1}$ & $\mathbf{5 1}$ & $\mathbf{5 2}$ & $\mathbf{5 3}$ & $\mathbf{5 4}$ & $\mathbf{5 5}$ \\
\hline
\end{tabular}

Tabela 9: Regras de cruzamento para obtenção do mapa final do potencial erosivo.

Table 9: Rule used to create the final erosive potential map.

\begin{tabular}{|c|c|}
\hline Potencial erosivo & Legenda \\
\hline Muito Estável & $\mathbf{1}$ \\
\hline Estável & $\mathbf{2}$ \\
\hline Potencialmente Instável & $\mathbf{3}$ \\
\hline Potencialmente M. Instável & $\mathbf{4}$ \\
\hline Instabilidade Emergente & $\mathbf{5}$ \\
\hline Instável & $\mathbf{6}$ \\
\hline Muito Instável & $\mathbf{7}$ \\
\hline
\end{tabular}

Tabela 10: Categorias da Carta de Fragilidade Ambiental das Unidades Ecodinâmicas.

Table 10: Classes of the erosive potential map.

\section{CONCLUSÃO E CONSIDERAÇÃO FINAL}

Apesar do tratamento utilizado ser considerado como qualitativo, pois o mesmo busca ordenar as diferentes regiões de acordo com regras de decisão, a metodologia adotada na obtenção do mapa do potencial erosivo pode ser considerada relativamente flexível e fácil de ser implementada, haja vista que as regras de cruzamento podem ser restabelecidas, a partir de diferentes visões de outros analistas. Uma outra abordagem seria transformar este estudo numa análise semi-quantitativa, baseada em probabilidades estimadas da influência de cada uma das variáveis na fragilidade ambiental. No entanto, esse tipo de estudo demandaria uma grande massa de dados empíricos.

Ainda, deve-se dizer que a metodologia empregada de tratamento e interpretação de imagens se mostrou eficaz e rápida, tendo em vista o propósito do mapa de uso e cobertura do solo.

Para refinamento e continuação deste trabalho, sugere-se a aplicação da equação universal de perda dos solos em cada uma das diferentes manchas de instabilidade/estabilidade. A partir desse resultado poder-se-á modelar as principais áreas fornecedoras de sedimentos aos maiores canais fluviais. Isto poderá ser implementado a partir da modelagem de escoamento superficial, usando-se do modelo digital de terreno e de operadores espaciais disponíveis em linguagens de álgebra cartográfica. Esses possibilitam não só identificar as microbacias de drenagem, como também traçar o curso de uma descarga de sedimento a partir de uma certa posição dentro da bacia hidrográfica (Cf. Usacerl, 1993).

\section{REFERÊNCIAS BIBLIOGRÁFICAS}

BERRY, J.K. 1993. Cartographic modeling: the analytical capabilities of GIS. In: GOODCHILD, M; PARKS, B.O. \& STEYAERT, L.T. (ed.) 1993. Environmental modelling with GIS. New York, Oxford University Press. cap.7, p.59-73.

BURROUGH, P.A. 1991. Principles of Geographical Information Systems for Land Resources Assessment. 3.ed. Oxford, Claredon Press, 230p.

EL-SWAIFY, S. A.; DANGLER; E. W. \& ARMSTRONG L. 1982. Soil erosion by water in the tropics. Department of Agronomy and Soil Science, College of Tropical Agriculture and Human Resources. US ISSN 0271-9916. Research Extension Series 024. University of Hawaii, Honolulu, Hawaii, 173 p.

JANSSON, M. B. 1982. Land erosion by water in different climates. Department of Physical Geography, Upsala University. UNGI Rapport no. 57. IBSN 91 - 506-0343-4, 151p.

MONMONIER, M. S. 1982. Computer-assisted cartography: principles and prospect. Prentice-Hall, New Jersey, $255 \mathrm{p}$.

ROSS, J. L. S. 1992. Análise empírica da fragilidade dos ambientes naturais e antropizados. Revista do Depto. de Geografia, FFLCHUSP, n. 6, São Paulo, 17-30.

ROSS, J. L. S. 1996. Geomorfologia aplicada aos EIAs-RIMAs. In: Guerra, A. J. T. \& Cunha, S. B. (ed.) 1996. Geomorfologia e meio ambiente. Bertrand/Brasil, São Paulo, p.291- 337.

TAYLOR, F. 1991. Geographic information systems: the microcomputer and modern cartography. Oxford, Pergamon Press, 230p.

TOMLIN, C. D. 1990. Geographic information systems and cartographic modeling. Prentice Hall, New Jersey, 249p.

TRICART, J. 1977. Ecodinâmica. FIBGE/SUPREN, Rio de Janeiro.

USACERL (United States Army Corps of Engineers Construction Engineering Research Laboratories) 1993. GRASS 4.1 User's reference manual. USACERL. Illinois, $340 \mathrm{p}$. 